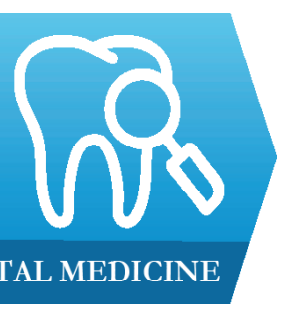

DENTAL MEDICINE

Department of Oral and Maxillofacial pathology, Vishnu Dental College, Bhimavaram, Andhra Pradesh, India

DOI: $10.15386 / \mathrm{mpr}-1746$

Manuscript received: 27.05.2020

Received in revised form: 01.07.2020

Accepted: 18.09.2020

Address for correspondence:

drswe82@gmail.com

This work is licensed under a Creative Commons Attribution-NonCommercialNoDerivatives 4.0 International License

\title{
Immunohistochemical evaluation of survivin in oral lichen planus and oral squamous cell carcinoma - a retrospective study
}

Tejaswi Lingam H, Swetha P, Ravikanth Manyam

\begin{abstract}
Introduction. Oral cancer is a multistep process involving enhanced activation of proto-oncogenes. Survivin (anti-apoptotic protein) is one of such proto-oncogenes that contribute to the carcinogenesis by deregulating cell proliferation. Expression of these proteins in cancerous and pre-cancerous lesions helps a better understanding of the etiology, treatment and prognosis.

Aim. To evaluate qualitative and quantitative expression of survivin in healthy mucosa, oral lichen planus (OLP) and oral squamous cell carcinoma (OSCC).

Methods. A total of 70 cases of formalin fixed paraffin embedded blocks, of which 20 healthy mucosa, 20 OSCC and 30 OLP were included in the study and stained immunohistochemically using antibodies against survivin monoclonal antibody. $4 \mu \mathrm{m}$ sections were stained immunohistochemically, both nuclear and cytoplasmic staining was considered as positive for surviving. Positive and negative stained cells were counted with the help of Olympus BX51 rarefaction microscope and were analyzed quantitatively using image analysis pro plus software. Representative fields were randomly selected at $40 \mathrm{X}$ high power magnification. Values obtained were evaluated and tabulated for statistical analysis.

Results. There was a statistically significant relation between the expressions of survivin among healthy mucosa, OLP, OSCC with a p value of 0.001 .

Conclusion. Presence of survivin expression in healthy mucosa, OLP and OSCC, which is an inhibitor of apoptosis protein, can be identified as a useful diagnostic tool for the identification of precancerous lesions and conditions which are at higher risk for progression into invasive carcinoma.
\end{abstract}

Keywords: carcinoma, healthy mucosa, OLP, OSCC, survivin, diagnostic tool

\section{Introduction}

Oral cancer accounts for approximately $3 \%$ of all malignancies world-wide [1]. Oral potentially malignant disorders (OPMDs) include a variety of lesions and conditions characterized by an increased risk for malignant transformation (MT) to oral squamous cell carcinoma (OSCC) $[2,3]$.

Among OPMDs, Oral Lichen Planus (OLP), constituting 0.5 to $2.2 \%$ of the general adult population, is the most common, chronic inflammatory, noninfectious oral mucosal disease [4]. OLPs are a mixture of white and red lesions that almost always present as bilateral symmetric pattern. White lesions have a reticular, papule, plaquelike appearance, and red lesions can appear atrophic (erythematous), erosive (ulcerated), or bullous-like. Lesions are not always homogenous; in some cases, they may present as a mixture of the above mentioned clinical subtypes.

White lesions generally form on a diffuse erythematous background however, reticular form shows a lace-like network of fine white lines known as Wickham's striae, whereas plaque forms appear as homogenous white patches resembling 
leukoplakia. The erosive form presents as atrophic and erythematous areas with partial ulceration, which are often surrounded by fine white lines. When erosion is severe, the epithelium ruptures, as in the case of benign mucous membrane pemphigoid. This type is known as bullous form and is very rare. The atrophic form appears as a diffuse red lesion with mucosal atrophy. The reported transformation rate varies from $0 \%$ to $9 \%$ with the erosive form being the most commonly associated clinical variant [5].

In general, malignant lesions are characterized by discrepancies in regulatory mechanisms controlling cell cycle progression, cell death and apoptosis [6]. Apoptosis is a genetically controlled process that plays a critical role in the differentiation and also in the elimination of senescent, unwanted and dangerous cells that sustain genetic damage or undergo uncontrolled cellular proliferation [7]. Inhibition of this process can lead to aberrantly reduced cell death and tumor genesis, also promoting increased resistance to therapy [8]. This evasion of apoptosis is the result of a disturbance of a group of proteins known as inhibitors of apoptosis proteins (IAPs).

However, histopathological features like epithelial dysplasia are the most potential indicators of malignant transformation where the assessment is done particularly based on morphological characters of the cells [3]. And yet, this alone does not provide an accurate assessment of malignant transformation, particularly where cells exhibit pleomorphism. Therefore, identifying a potential predictor in OPMDs facilitates prevention and early diagnosis of carcinoma.

Survivin is one such potential predictor of the IAP's expressed in most of the tumors. Survivin functions to inhibit caspase activation, thereby leading to negative regulation of apoptosis. Survivin exists in two subcellular levels (cytoplasmic and nuclear), cytoplasmic participates in controlling cell survival but not cell proliferation, while nuclear level is involved in promoting cell proliferation $[9,10]$. It has been reported that elevated expression of survivin is associated with enhanced cell proliferation, reduced levels of apoptosis, resistance to chemotherapy and increased rate of recurrence.

Expression of survivin protein in OSCC has been noted to play a vital role in oral carcinogenesis and is established as a diagnostic tool [11,12]. So the use of survivin in OLP and OSCC could be useful as an early predictor for malignant transformation. Hence, this study aims to evaluate qualitatively and quantitatively the expression of survivin in OLP and OSCC.

\section{Methods}

\section{Study design}

This is a retrospective study conducted for the immunohistochemical (IHC) evaluation of survivin in OLP and OSCC. Specimens included 70 histopathologically diagnosed, formalin fixed paraffin embedded blocks of 20 normal oral mucosa, 30 OLP and 20 OSCC from the archives of Department of Oral Pathology, Vishnu Dental College. Specimens were further segregated based on age, gender, habit history and lesion site from the records. Institutional ethical clearance was obtained from the institute before the commencement (VDC/IEC/2016/70).

\section{Procedure}

\section{Step 1. Preparation of tissue sections}

Formalin fixed paraffin embedded tissues were sectioned at $4 \mu \mathrm{m}$ and mounted on positively charged slides. These slides were incubated at $60^{\circ} \mathrm{C}$ for $1 \mathrm{hr}$ and de-paraffined using two different xylene solutions for 10 minutes. Later rehydrated with $100 \%$ alcohol for 10 minutes and $80 \%$ alcohol for another 10 minutes and were washed with distilled water.

Step 2. Antigen retrieval

Antigen retrieval was done using pressure cooker method by preheating the buffer (2.94 gms of tri sodium citrate in $1000 \mathrm{ml}$ of distilled water) for 3-5 minutes then slides were treated with buffer solution. Slides were washed with phosphate buffered saline (PBS) twice each for 5 minutes. Before the sections were incubated with antibodies, all the sections were encircled with PAP pen and peroxide block was added for 10 minutes and washed with PBS. Then the power block was added for 10 minutes and then it was washed with PBS.

These slides were incubated with primary antibody for 1 hour and then washed with PBS followed by addition of primary antibody enhancer for 20 minutes and washed with PBS. Later these slides were incubated with secondary antibody Poly HRP for 30 minutes and washed with PBS for 5 minutes.

Finally, prior to counterstaining, slides were incubated with DAB substrate chromogen for 3 minutes and washed in distilled water for 5 minutes and counterstained with Harris haematoxylin for 3 minutes followed by bluing in running tap water. Slides were dried and dehydrated in absolute alcohol for 3 minutes followed by xylene for 5 minutes and mounted with DPX.

\section{Step 3. Evaluation of staining intensity}

Ten representative fields were randomly selected and observed for quantification and localization of stain at 40X high power magnification and were graded based on their distribution as follows:

- Focal - Positive cells Less than 50\% when viewed under lower magnification.

- Diffuse - Positive cells more than 50\% when viewed under lower magnification.

Grading of Qualitative and Quantitative expression of antigen was done as follows:

Qualitative Analysis:

The staining intensity was classified as: 0-No staining; 1-Weak; 2-Moderate; 3-Strong.

Quantitative Analysis:

The staining extent was classified as: 
0 - Less than $25 \%$ of epithelial cells demonstrated positivity;

$1-25 \%$ to $50 \%$ of cells demonstrated positivity;

$2-50 \%$ to $75 \%$ of cells demonstrated positivity;

3 - Greater than $75 \%$ of cells demonstrated positivity.

A final score was established by adding the two indices and were graded into four groups:

0 - Negative, 1-3 - Weak, 4-5 - Moderate, 6-7 Strong.

\section{Results}

A total of 70 specimens were examined for the presence of antigen survivin and graded as mentioned and subjected for statistical analysis using SPSS software. Pearson Chi-square test was used for comparing the staining pattern, intensity and localization of the survivin in study groups. One-way ANOVA test was used for comparing average number of positive cells in different study groups.

All the specimens were categorized based on age and gender (Table I). On intragroup comparison, the expression of survivin in healthy tissues was focally distributed whereas in OLP and OSCC survivin expression was predominantly diffuse in nature with statistical significance (Table II).

Table I. Distribution of percentage of study population in normal tissues.

\begin{tabular}{l|c|c|c|c|}
\multirow{2}{*}{ Age/ Gender } & \multirow{2}{*}{ Category } & \multicolumn{3}{|c|}{ Group } \\
\cline { 3 - 5 } & & $\begin{array}{c}\text { Healthy } \\
\text { Mucosa }\end{array}$ & OLP & OSCC \\
\hline \multirow{2}{*}{ Age $(\mathrm{n}=70)$} & $0-20$ & $10(50 \%)$ & $6(20.0 \%)$ & $0(0 \%)$ \\
& $20-41$ & $4(20 \%)$ & $16(53.3 \%)$ & $14(70 \%)$ \\
Gender & $41-65$ & $6(30 \%)$ & $8(26.7 \%)$ & $6(30 \%)$ \\
$(\mathrm{n}=70)$ & male & $7(35 \%)$ & $12(40 \%)$ & $16(80 \%)$ \\
& female & $13(65 \%)$ & $18(60 \%)$ & $4(20 \%)$
\end{tabular}

Table II. Comparison of staining distribution pattern for survivin within groups.

\begin{tabular}{l|c|c|c|c|c|} 
Specimen & \multirow{2}{*}{ No. } & \multicolumn{2}{|c|}{ Distribution } & Chi-square & P-value \\
\cline { 3 - 5 } & & Focal & Diffuse & value & \\
\hline $\begin{array}{l}\text { Healthy } \\
\text { Mucosa }\end{array}$ & 20 & 13 & 00 & & \\
OLP & 30 & 03 & 27 & 58.76 & 0.00 \\
OSCC & 20 & 00 & 20 & & \\
\end{tabular}

Healthy mucosa exhibited weak positivity (13 sections) in the basal layers whereas 20 sections of OLP exhibited moderate staining in the basal layer, 7 sections expressed intense staining within the basal layers and 3 sections expressed moderate staining within the superficial layers. OSCC (18 cases) showed severe staining intense in the superficial layers, and 2 cases showed strong intense staining within basal layers (Table III) (Figure 1, Figure 2, Figure 3).
Table III. Comparison of intensity of survivin in different layers of control group and study groups.

\begin{tabular}{ll|c|c|c|c|c} 
& Layers & N & Weak & Moderate & Strong & p value \\
\hline Healthy & Basal & 13 & 3 & 10 & 0 & 0.00 \\
Mucosa & Superficial & 0 & 0 & 0 & 0 & \\
\multirow{2}{*}{ OLP } & Basal & 27 & 0 & 20 & 7 & 0.00 \\
& Superficial & 3 & 0 & 3 & 0 & \\
\multirow{2}{*}{ OSCC } & Basal & 2 & 0 & 0 & 2 & 0.00
\end{tabular}

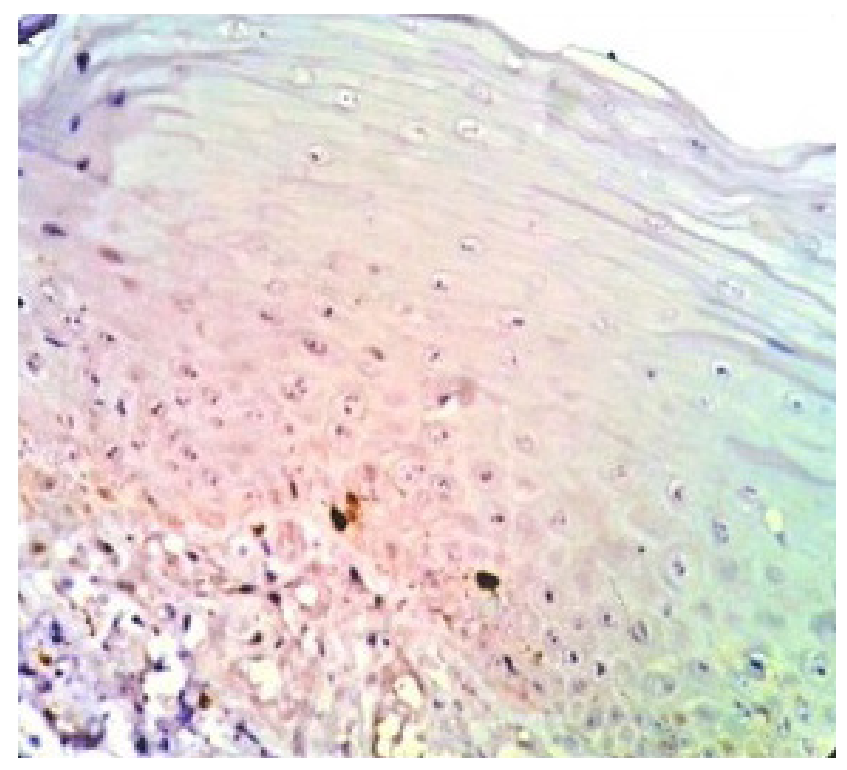

Figure 1. Focal and weak staining in the basal layer of healthy mucosa.

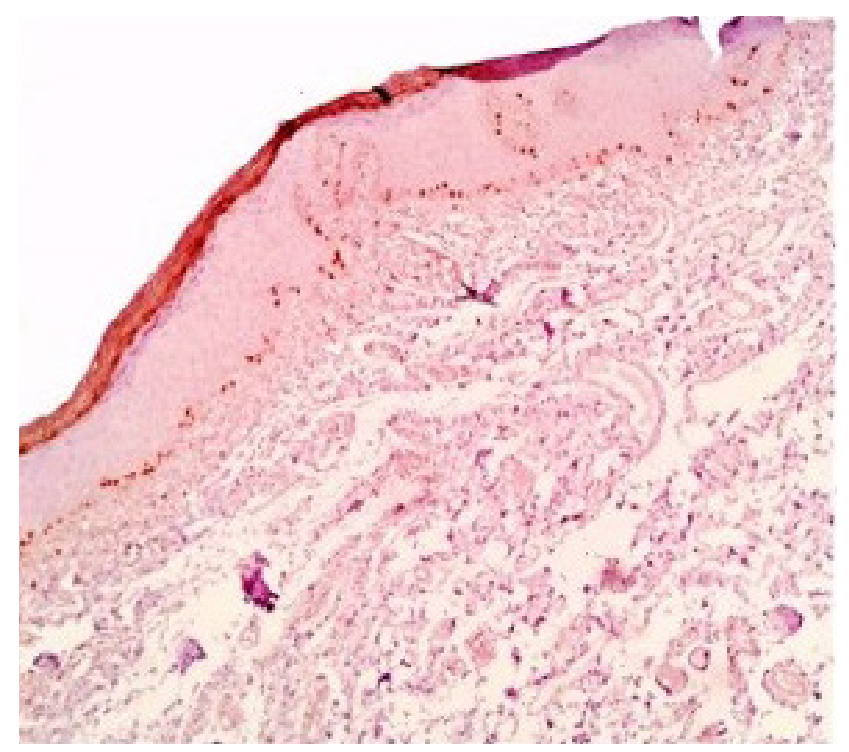

Figure 2. Diffuse and moderate staining in OLP. 


\section{Dental Medicine}

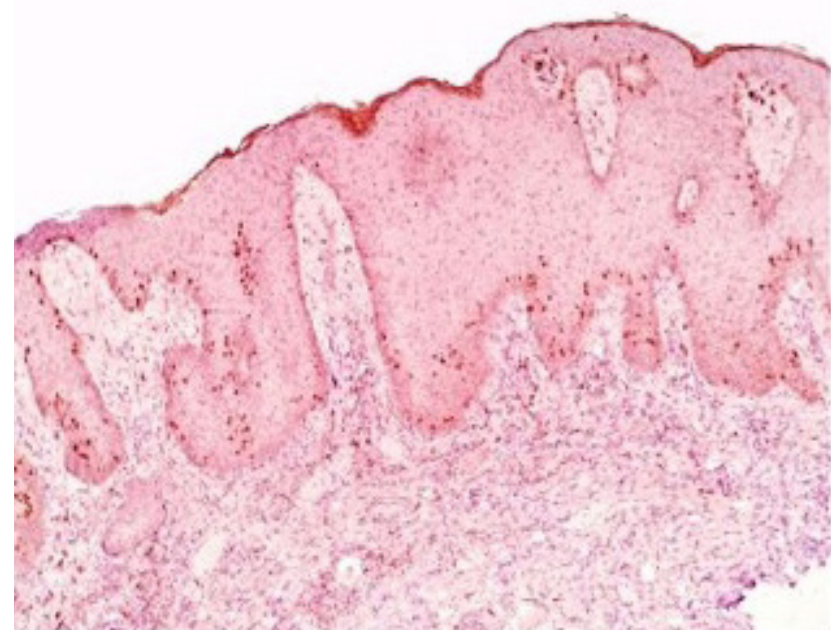

Figure 3. Diffuse and strong intensity of survivin staining in the basal and suprabasal layer of OSCC.

However, distribution of survivin among the different types of OLP (30 cases), i.e. reticular type (13), erosive type (8) and plaque type OLP (6) exhibited diffuse staining, whereas reticular type (2) and plaque type (1) exhibited focal distribution of staining. (Table IV) (Figure 4, Figure 5).

Table IV. Comparison of staining distribution pattern for survivin within OLP groups.

\begin{tabular}{|l|c|c|c|c|}
\hline \multirow{2}{*}{ Types of OLP } & \multicolumn{2}{|c|}{ Distribution } & Chi-square & \multirow{2}{*}{ P-value } \\
\cline { 2 - 3 } value & Focal & Focal & & \\
\hline Reticular OLP & 2 & 3.016 & & \\
Plaque OLP & 1 & 1 & 0.221 & 0.00 \\
Erosive OLP & 0 & 0 & & \\
\hline
\end{tabular}

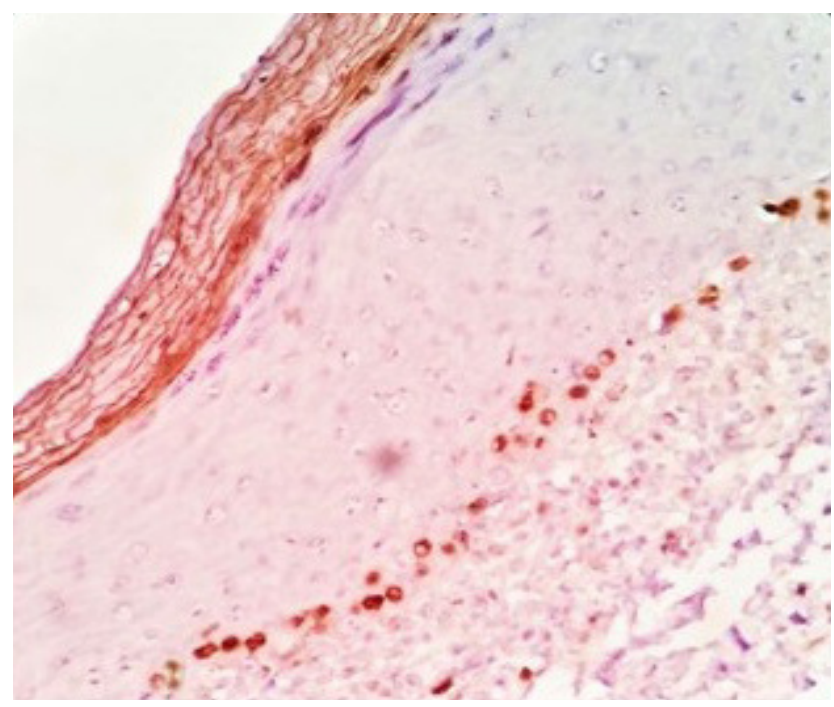

Figure 4. Nuclear positivity of survivin predominantly in the basal layer of the plaque variant of OLP.

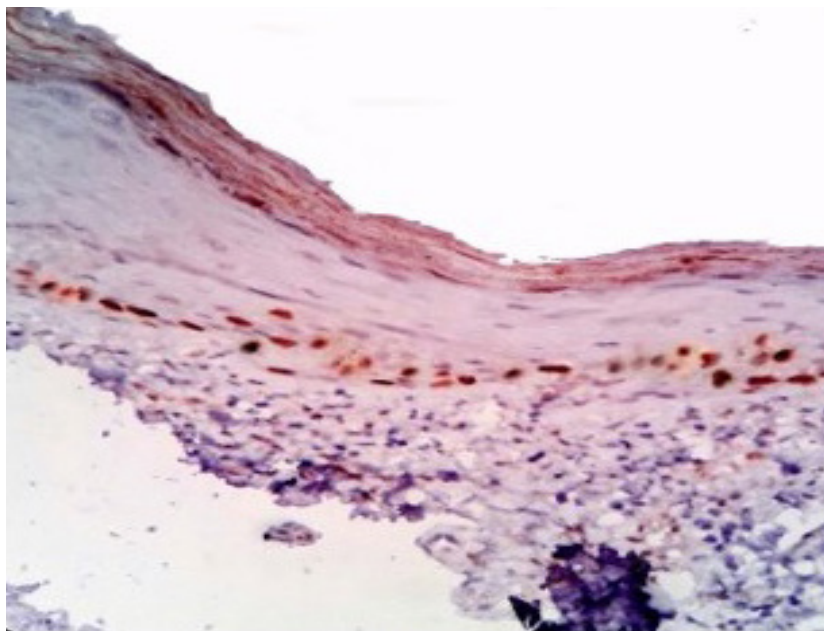

Figure 5. Nuclear positivity of survivin predominantly in the basal and suprabasal layer of the erosive variant of OLP.

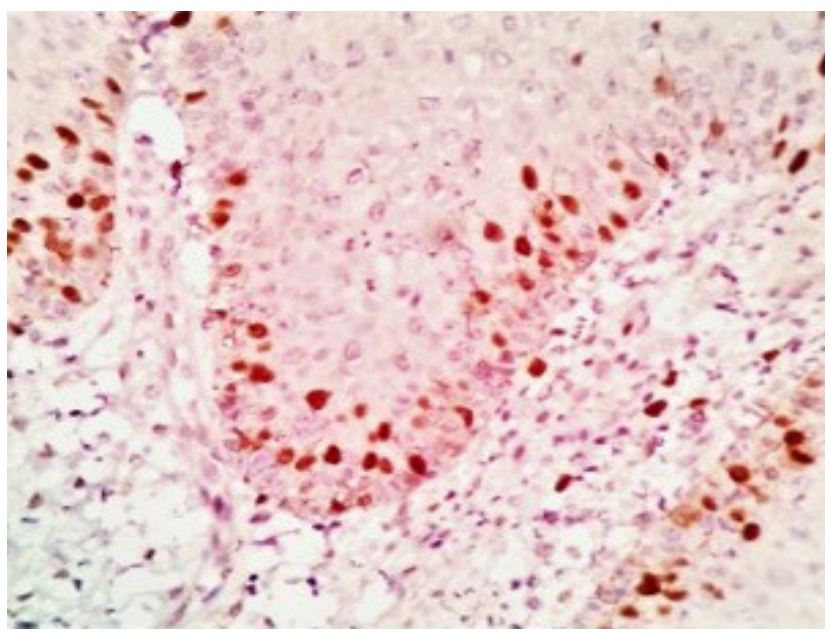

Figure 6. Nuclear positivity of survivin predominantly in the basal and suprabasal layer of well differentiated OSCC.

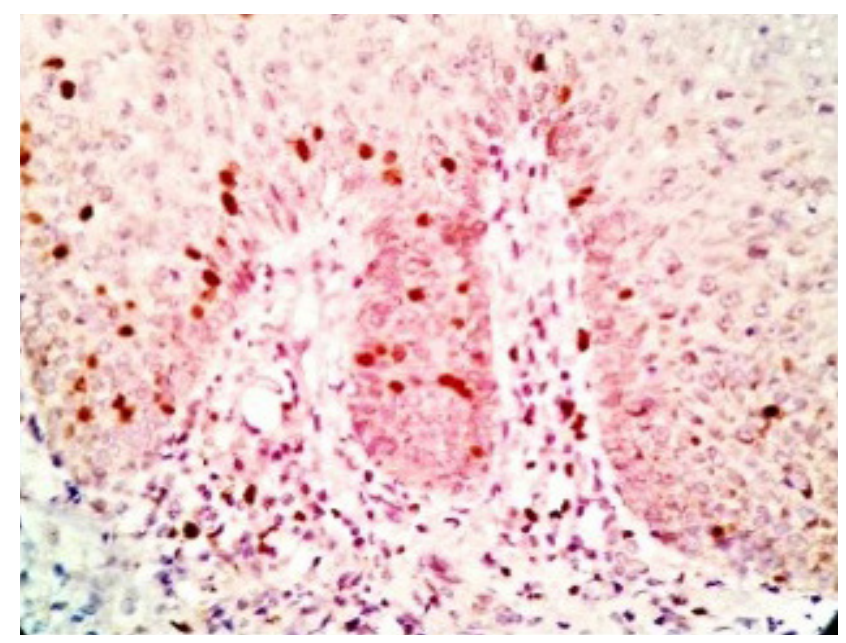

Figure 7. Nuclear and cytoplasmic positivity of survivin in the basal and suprabasal layer of moderately differentiated OSCC. 
There is a predominant nuclear positivity, seen in all grades of OSCC, with some cases showing both cytoplasmic and nuclear distribution of survivin (Figure 6, Figure 7).

\section{Discussion}

OSCC (90\%) is the utmost malignant tumor of the oral cavity. In spite of several preventive measures and advancements, use of tobacco and alcohol play a vital role, and the 5 -year survival time remains about $55 \%$ of the cases [13-16]. When the steadiness between proliferation and apoptosis is altered, there will be transition of normal epithelium to dysplastic epithelium. These alterations may be accompanied by "multiple hits" (i.e. Knutson hypothesis) promoting proliferation, angiogenesis, local invasion and eventually distant metastasis [17].

The malignant process involves DNA damage during its replication or by deficiencies of its repair. These DNA alterations usually occur in genes that regulate the cell cycle, growth and differentiation. Oncogenes are originated from proto-oncogenes, which are involved in basic mechanisms of normal cell growth regulation and include growth factors, receptors for growth factors, signal transducers and nuclear transcription factors [17].

Oral Potenially Malignant Disorder (3-33\%) will evolve into invasive OSCC over a 10 years' interval, no reliable histopathological parameters have been identified that predict their potential for subsequent transformation. Novel molecular predictors of malignant progression are needed to identify OPMDs at greater risk of invasive transformation [13].

Several molecular markers implicated in the carcinogenesis of OSCC have been evaluated by many investigators, including molecules involved in the cell cycle regulation, apoptosis, angiogenesis, DNA repair system and degradation of extracellular matrix. Identifying these molecules by analysis of DNA, RNA and protein expression might permit the early diagnosis. The most used techniques have been cDNA microarray, PCR, in situ hybridization, immunohistochemistry (IHC), ELISA and western-blot. However, the available evidence about the molecular markers still remains inconclusive. Accordingly, there is a continuous need to define the most important biological markers for OSCC progression and invasiveness [14].

Much importance has been given to apoptotic markers that are expressed in both premalignant and malignant lesions. Among them, survivin is a recently characterized protein marker which is found and expressed in solid and hematological malignancies [13]. A unique member in the IAP (inhibitor of apoptosis protein) family, it is undetectable in healthy adult tissues but is highly expressed in cancer. It is cell cycle regulated protein, and is involved in both control of apoptosis (or programmed cell death) as well as regulator of cell division [18-21].

Further investigation of survivin and other apoptotic inhibitors during tumor growth and progression may yield important therapeutic strategies for fighting cancer. Therefore, the present study was designed to analyze the immunohistochemical expression of survivin in OMPD such as OSCC and OLP.

In our study, OSCC exhibited male predominance with male to female ratio (4:1), whereas there was female predominance with male to female ratio (1:3) in OLP cases. Documentation of the associated habits and common site of the lesion showed tobacco as the most prevalent cause for squamous cell carcinoma and no habit relation in OLP. However, buccal mucosa was the most favorable site of occurrence in OLP and the vestibule is the most favorable site of occurrence in the OSCC.

Several studies have reported an absence of survivin expression in normal oral mucosal tissue specimens [13,22]. On the other hand, Negi at el. have reported survivin positivity in almost 20 percent of normal tissue specimens and attributed this to higher proliferative capacity and pointed out that the survivin immune expression in normal oral epithelium is due to the presence of active mitotic cells $[22,23]$. It was stated that survivin was present in normal hematopoietic cells, immune cells, vascular endothelial cells, polymorphonuclear neutrophils, $\mathrm{T}$ lymphocytes, melanocytes, keratinocytes, neurons, and other cells of the mammalian brain [24].

A study by Chaiyarit and others demonstrated significant expression of survivin in normal oral epithelial cells was due to processing errors in fixation, antigen retrieval techniques, types of primary antibodies and detecting systems [24]. In our study survivin positivity in normal mucosa was noted in 13 cases. The staining distribution pattern was focal and weak in intensity limiting to basal layer. The total positivity rate was only $0.26 \%$ when compared to negative expression (99\%). The results of this study were in accordance with the studies reported by Lo Muzio et al. and Tanaka et al [13].

Earlier studies demonstrated survivin expression in OLP to be $95 \%$ which was mainly of moderate intensity, followed by mild intensity with predominant survivin expression in the basal layer of cells and all the cells showed $100 \%$ nuclear positivity [12]. In our study, the majority of the cases expressed mild to moderate intensity with nuclear expression of survivin primarily in the basal layer of the epithelium and are in accordance with the study by Suganya et al [12]. Chaiyarit et al., demonstrated survivin expression in the nucleus predominantly with one fourth of cases demonstrating both nuclear and cytoplasmic positivity in OLP [24]. Despite the fact that nuclear expression of survivin is an unfavorable factor for prognosis in various tumors and OPMDs occurring in humans, some authors have proposed survivin nuclear positivity as a favorable prognostic marker [10].

Survivin can travel between the nucleus and cytoplasm and it plays a cyto-protective role function by 
facilitating survivin interplay with the apoptotic machinery in cancer cells within the cytoplasm and likewise play a role in cell division within the nucleus. This bi-functional role of survivin is dependent on various factors like nuclear export receptors, chromosomal passenger complexes and basic amino acids [25].

In this study, among the various types of OLP, the majority of the cases showed diffuse staining pattern. The intensity of the staining is moderate and is confined to basal layers in reticular and plaque variants whereas in most of the cases of erosive lichen planus the staining intensity is strong and also seen in suprabasal layers. However, survivin levels were highest in erosive forms of OLP when compared to reticular and plaque forms. Therefore, the erosive form of OLP may be considered as an active condition with high chances of malignant transformation. On the other hand, reticular and plaque forms of OLP can be considered as a much stable condition with decreased chances of malignant transformation.

Survivin positivity in OSCC cell lines in hamster oral carcinogenesis model expressed $100 \%$ and showed that OSCC patients with high survivin expression in the tumor mass had a shorter survival [26]. Likewise many other studies have reported survivin presence in higher rates $[13,22,27,28]$.

In our study, out of 20 cases of OSCC there were 10 cases of well differentiated and 10 cases of moderately differentiated exhibited diffuse staining pattern expression. The intensity of the staining is strong and is confined to both basal and supra basal layers. The overall survivin positivity in oral squamous cell carcinoma was $100 \%$ and was in accordance with several studies $[13,29,30]$. In the present study grades of survivin expression is increased from well differentiated to moderately differentiated OSCC.

The present study reported a predominant nuclear positivity seen in all grades of OSCC with a few cases exhibited both cytoplasmic and nuclear distribution of survivin. Well differentiated OSCC showed nuclear staining for survivin, while moderately and poorly differentiated OSCC exhibited both nuclear and cytoplasmic staining. However, cytoplasmic expression was found be prevalent in poorly differentiated cases and prominent nuclear staining in well-differentiated areas in the study conducted by Muzio et al.

Survivin expression when compared among normal oral mucosa, leukoplakia and OSCC there was statistically significant difference in number of survivin positive cells (value $<0.001$ ), indicating that overexpression of survivin is associated with uncontrolled cell growth [31].

The tumor suppressor gene p53 which is called as "guardian of genome," plays a critical role in the maintenance of genomic integrity by controlling the cell cycle, DNA repair, and activation of apoptosis. Aberrant expression of this gene or mutant $\mathrm{p} 53$ has been implicated in the development and progression of oral epithelial dysplasia and oral squamous cell carcinoma. It has been well documented that the expression of survivin is downregulated by $\mathrm{p} 53$, and the repression of survivin is tightly related to p53-dependent apoptosis $[32,33]$. In the present study the increased expression of survivin levels in OSCC might be due to impaired function of p53 gene resulting in evasion of apoptosis and uncontrolled cell growth.

\section{Summary}

To summarize, in order to achieve a molecular targeted approach in diagnosis, prognosis and therapy, histopathology needs to be complemented with reliable molecular parameters. This remains a valued and viable diagnostic tool. OLP lesions that are in the process of transforming into OSCC will possess certain molecular signatures which will provide critical information on disease entities and also further clarify the potential progression of OLP to OSCC. Therefore, it is advisable to monitor the progression of the disease both histopathologically as well as at a molecular level. Use of biomarker survivin in OPMDs such as OLP may provide advantage in early diagnosis, assessment and prognosis of OLP. Survivin expression together with its cellular localization should be considered to evaluate its impact on future prognosis and therapy options.

\section{Conclusion}

It can be concluded that the diagnosis of OLP histopathologically together with molecular profiling and localization of survivin levels can be used as an early biomarker for malignant transformation.

\section{Limitations}

Large study samples are needed to corroborate the prognostic impact of survivin expression in OPMDs and OSCC, however our findings suggest that survivin detection may provide a new tool to identify the patients with OPMDs that are at high risk for malignant transformation.

\section{Acknowledgement}

I would like to acknowledge the statistician for conducting the analysis.

\section{References}

1. Mortazavi H, Baharvand M, Mehdipour M. Oral potentially malignant disorders: an overview of more than 20 entities. J Dent Res Dent Clin Dent Prospects. 2014;8:6-14.

2. Ho PS, Chen PL, Warnakulasuriya S, Shieh TY, Chen YK, Huang IY. Malignant transformation of oral potentially malignant disorders in males: a retrospective cohort study. BMC Cancer. 2009;9:260.

3. Farah CS, Woo SB, Zain RB, Sklavounou A, McCullough MJ, Lingen M. Oral cancer and oral potentially malignant disorders. Int J Dent. 2014;2014:853479. 
4. Ion DI, Setterfield JF. Oral Lichen Planus. Prim Dent J. 2016;5:40-44.

5. Bandyopadhyay A, Behura SS, Nishat R, Dash KC, Bhuyan L, Ramachandra S. Clinicopathological Profile and Malignant Transformation in Oral Lichen Planus: A Retrospective Study. J Int Soc Prev Community Dent. 2017;7:116-124.

6. Wong RS. Apoptosis in cancer: from pathogenesis to treatment. J Exp Clin Cancer Res. 2011;30:87.

7. Elmore S. Apoptosis: a review of programmed cell death. Toxicol Pathol. 2007;35:495-516.

8. Hassan M, Watari H, AbuAlmaaty A, Ohba Y, Sakuragi N. Apoptosis and molecular targeting therapy in cancer. Biomed Res Int. 2014;2014:150845.

9. Garg H, Suri P, Gupta JC, Talwar GP, Dubey S. Survivin: a unique target for tumor therapy. Cancer Cell Int. 2016; 16:49.

10. Li F, Yang J, Ramnath N, Javle MM, Tan D. Nuclear or cytoplasmic expression of survivin: what is the significance? Int J Cancer. 2005;114:509-512.

11. Liu XZ, Li CJ, Wu SJ, Shi X, Zhao JN Involvement of $\alpha 5$ integrin in survivin-mediated osteosarcoma metastasis. Asian Pac J Trop Med. 2016;9:478-483.

12. Suganya G, Bavle RM, Paremala K, Makarla S, Sudhakar M, Reshma V. Survivin expression in oral lichen planus: Role in malignant transformation. J Oral Maxillofac Pathol. 2016;20:234-238.

13. Lo Muzio L, Pannone G, Leonardi R, Staibano S, Mignogna MD, De Rosa G, et al. Survivin as a potential early predictor of tumor progression in the oral mucosa. J Dent Res. 2003;82:923-928.

14. Daniel FI, Fava M, Hoffmann RR, Campos MM, Yurgel LS. Main Molecular Markers of Oral Squamous Cell Carcinoma. Applied Cancer Research 2010; 30(3): 279-288.

15. Bannerjee AG, Bhattacharyya I. Identification of genes and molecular pathways involved in the progression of premalignant oral epithelia. Mol Cancer Ther. 2005;4:865875.

16. Prakin DM, Bray F, Ferlay J, Pisani P. Global cancer statistics, 2002. CA Cancer J Clin. 2005;55:74-108.

17. Tsantoulis PK, Kastrinakis NG, Tourvas AD, Laskaris G, Gorgoulis VG. Advances in the biology of oral cancer. Oral Oncol. 2007;43:523-534.

18. Li F. Role of survivin and its splice variants in tumorigenesis. Br J Cancer. 2005;92:212-216.

19. Chiou SK, Jones MK, Tarnawski AS. Survivin an antiapoptosis protein: its biological roles and implications for cancer and beyond. Med Sci Monit. 2003;9:PI25-PI29.

20. Dallaglio K, Marconi A, Pincelli C. Survivin: a dual player in healthy and diseased skin. J Invest Dermatol. 2012;132:1827.

21. Fukuda S, Pelus LM. Survivin, a cancer target with an emerging role in normal adult tissues. Mol Cancer Ther. 2006;5:1087-98.

22. Tanaka C, Uzawa K. Expression of an inhibitor of apoptosis, surviving, in oral carcinogenesis. J Dent Res. 2003;82:607611.

23. Negi A, Puri A, Gupta R, Nangia R, Sachdeva A, Mittal M. Comparison of Immunohistochemical Expression of Antiapoptotic Protein Survivin in Normal Oral Mucosa, Oral Leukoplakia, and Oral Squamous Cell Carcinoma. Patholog Res Int. 2015;2015:840739.

24. Chaiyarit P, Jintakanon D, Klanrit P, Siritapetawee M, Thongprasom K. Immunohistochemical analyses of survivin and heat shock protein 90 expression in patients with oral lichen planus. J Oral Pathol Med. 2009;38:55-62.

25. Stauber RH, Mann W, Knauer SK. Nuclear and cytoplasmic survivin: molecular mechanism, prognostic, and therapeutic potential. Cancer Res.2007;67:5999-6002.

26. Kim YH, Kim SM, Kim YK, Hong SP, Kim MJ, Myoung $\mathrm{H}$. Evaluation of survivin as a prognostic marker in oral squamous cell carcinoma. J Oral Pathol Med. 2010;39:368375.

27. Lo Muzio L, Staibano S, Pannone G, Mignogna MD, Mariggiò A, Salvatore G, et al. Expression of the apoptosis inhibitor survivin in aggressive squamous cell carcinoma. Exp Mol Pathol. 2001;70:249-254.

28. Jinbu Y, Tsukinoki K, Miyagi N, Senna T, Obi Y, Matsumoto $\mathrm{K}$, et al. Expression of survivin in oral squamous cell carcinoma. Oral Med Pathol. 2006;11:41-44.

29. Oluwadara O, Giacomelli L, Christensen R, Kossan G, Avezova R, Chiappelli F. LCK, survivin and PI-3K in the molecular biomarker profiling of oral lichen planus and oral squamous cell carcinoma. Bioinformation. 2009;4:249-257.

30. Lin CY, Hung HC, Kuo RC, Chiang CP, Kuo MY. Survivin expression predicts poorer prognosis in patients with areca quid chewing-related oral squamous cell carcinoma in Taiwan. Oral Oncol. 2005;41:645-654.

31. Hoffman WH, Biade S, Zilfou JT, Chen J, Murphy M. Transcriptional repression of the anti-apoptotic survivin gene by wild type p53. J Biol Chem 2002;277:3247-3257.

32. Mirza A, McGuirk M, Hockenberry TN, Wu Q, Ashar H, Black $\mathrm{S}$, et al. Human survivin is negatively regulated by wild-type p53 and participates in p53-dependent apoptotic pathway. Oncogene. 2002;21:2613-2622.

33. Jin Y, Wei Y, Xiong L, Yang Y, Wu JR. Differential regulation of survivin by p53 contributes to cell cycle dependent apoptosis. Cell Res. 2005;15:361-370. 